\title{
A photospheric bright point model
}

\author{
S. Shelyag, M. Mathioudakis, F. P. Keenan, and D. B. Jess
}

Astrophysics Research Centre, School of Mathematics and Physics, Queen's University, Belfast, BT7 1NN, Northern Ireland, UK

Received 10 December 2009 / Accepted 8 March 2010

\begin{abstract}
Aims. A magneto-hydrostatic model is constructed with spectropolarimetric properties close to those of solar photospheric magnetic bright points.

Methods. Results of solar radiative magneto-convection simulations are used to produce the spatial structure of the vertical component of the magnetic field. The horizontal component of magnetic field is reconstructed using the self-similarity condition, while the magneto-hydrostatic equilibrium condition is applied to the standard photospheric model with the magnetic field embedded. Partial ionisation processes are found to be necessary for reconstructing the correct temperature structure of the model.

Results. The structures obtained are in good agreement with observational data. By combining the realistic structure of the magnetic field with the temperature structure of the quiet solar photosphere, the continuum formation level above the equipartition layer can be found. Preliminary results are shown of wave propagation through this magnetic structure. The observational consequences of the oscillations are examined in continuum intensity and in the Fe I $6302 \AA$ magnetically sensitive line.
\end{abstract}

Key words. Sun: oscillations - Sun: photosphere - Sun: surface magnetism - plasmas - magnetohydrodynamics (MHD) radiative transfer

\section{Introduction}

The study of magnetic elements at very small scales is one of the most important topics in solar physics. Magnetic bright points (MBPs) are ubiquitous in the solar photosphere. They have small diameters, typically less than $300 \mathrm{~km}$, and are found in the intergranular lanes. MBPs correspond to areas of kilogauss fields, are best observed in G-band disk centre images and are numerous in active regions or near sunspots. They are formed by a complex process involving the interaction of the magnetic field with the convectively unstable hot plasma. The physical processes associated with their formation have been outlined in Schüssler et al. (2003); Shelyag et al. (2004); Carlsson et al. (2004), using forward modelling of radiative magneto-convection in the solar photosphere and upper convection zone.

Despite the overall success of photospheric and subphotospheric radiative magneto-convective models to reproduce many of the observational properties of solar radiation, we still do not fully understand the physical processes involved in the strongly magnetised photospheric plasma. In particular, it is difficult to use the results of these simulations for studies of acoustic wave propagation through the solar atmosphere and interior. Strong convective motions of the photospheric plasma can hide the signatures of acoustic waves, making them a difficult subject in both numerical and observational investigations.

The development of new methods for inferring the properties of solar plasma using sound waves have been followed by the successful modelling of the magneto-acoustic properties in the solar atmosphere and interior (Hasan et al. 2005; Hanasoge et al. 2007; Hasan \& van Ballegooijen 2008; Fedun et al. 2009; Khomenko et al. 2009; Parchevsky \& Kosovichev 2009; Shelyag et al. 2009; Steiner 2009; Vigeesh et al. 2009). However, due to the non-locality of radiative processes in the solar atmosphere, a direct comparison of the plasma parameters at a certain geometrical depth in the computational box with the solar radiation parameters at a given optical depth may not be entirely correct. The non-locality of radiative transport must be taken into account. Khomenko \& Collados (2009) have recently suggested that the changes in the height of continuum formation with respect to the equipartition layer (the layer where the Alfvén speed is equal to the sound speed, $v_{\mathrm{A}}=c_{\mathrm{S}}$ ), may help to explain the appearance of the high-frequency acoustic haloes around sunspots.

Numerical simulations of solar wave phenomena require a static magnetic configuration model which incorporates as many physical properties of the real Sun as possible. In this paper we provide a recipe to create such a model, based on the results of numerical modelling of magneto-convection in the photosphere. We demonstrate that the spectropolarimetric properties of the magnetic configuration we created successfully reproduces those of MBPs. In Sect. 2 we describe the technique used to reconstruct the magnetic and thermal parameters of the MBP model. The results of the spectropolarimetric simulations using the model are presented in Sect. 3. In Sect. 4 we show our preliminary results on the wave mode conversion in the MBP and discuss the possible observational signatures. Our concluding remarks are presented in Sect. 5.

\section{Static bright point model}

We use a snapshot from the "plage" magneto-convection simulation of the solar photosphere undertaken with the MURaM code (Vögler et al. 2005) to produce the average MBP model. Since the average magnetic field flux in this snapshot is relatively high $(200 \mathrm{G})$, a large number of intergranular magnetic field concentrations are generated. The magnetic field concentrations appear bright in the continuum $(\lambda=4300 \AA)$, and in the $\mathrm{G}$ band. This snapshot has been used to demonstrate the magnetic nature of the G-band bright points (Schüssler et al. 2003; Shelyag et al. 2004). To reveal the basic structure of the vertical component of the magnetic field $B_{0 z}$ in the MBPs, we average the 


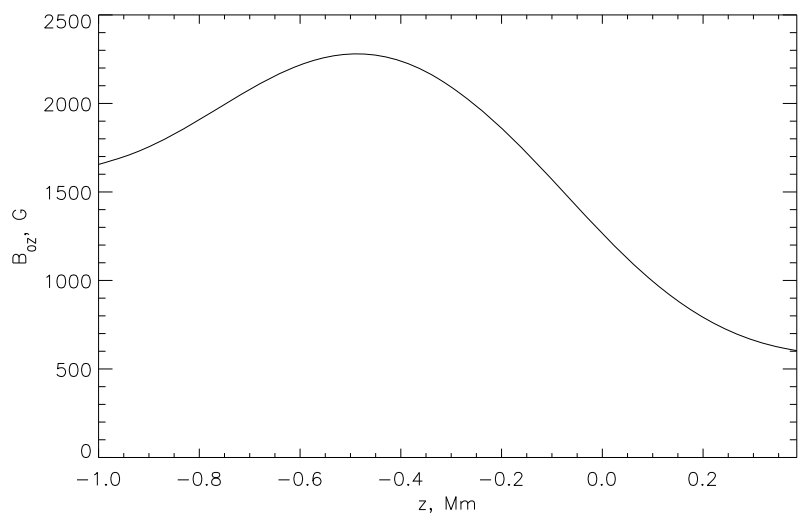

Fig. 1. Vertical magnetic field component $B_{0 z}$ as a function of depth for the photospheric MBPs. A depth level of $z=0$ corresponds to $\log \tau_{4300}=0$ in the quiet solar model.

depth dependences of the vertical magnetic field strength over the bright points, which are selected by their enhanced G-band intensity and magnetic field strength. Figure 1 shows $B_{0 z}$ as a function of depth. Note that $z=0$ on the depth scale corresponds to the average optical depth $\log \tau_{4300}=0$ for the quiet Sun, and thus does not include Wilson depression (Wilson \& Maskelyne 1774). The Wilson depression is about $100 \mathrm{~km}$, as will be shown later.

We use the self-similarity assumption (Schlüter \& Temesváry 1958; Schüssler \& Rempel 2005; Gordovskyy \& Jain 2007; Shelyag et al. 2009) to reconstruct the horizontal component of the magnetic field. Below we briefly discuss its governing equations.

For the prescribed vertical magnetic field component $B_{0 z}(z)$, defined along the axis of the symmetric magnetic field configuration, the (divergence-free) two-dimensional magnetic field can be defined as:

$B_{z}(x, z)=B_{0 z} f\left(x B_{0 z}\right)$

and

$B_{x}(x, z)=-\frac{\partial B_{0 z}}{\partial z} x f\left(x B_{0 z}\right)$,

where $f$ is an arbitrary function which describes how the vertical component of the field expands with height.

For the simulations, we choose the following grid parameters of the numerical domain in which the magnetic field is embedded: the vertical and horizontal extents of the domain are $1.4 \mathrm{Mm}$ and $2 \mathrm{Mm}$, respectively, which are resolved in $100 \times 200$ grid cells.

In Fig. 2 we show the magnetic field configuration calculated for this domain using the above definition. The function $f$ takes zero values on the side boundaries of the domain and is chosen in such a way that the radius of the magnetic field region is approximately $200 \mathrm{~km}$ at the height corresponding to the quiet Sun continuum formation level.

The magnetic field changes the thermodynamic parameters of the plasma where it is embedded. These changes can be quite significant for the plasma dynamics and wave propagation (see e.g. Shelyag et al. 2009). To construct the magneto-hydrostatic configuration, we substitute the values of the magnetic field components obtained using Eqs. (1) and (2) into the equation of magneto-hydrostatic equilibrium

$(\boldsymbol{B} \cdot \nabla) \boldsymbol{B}+\nabla \frac{B^{2}}{2}+\nabla p=\rho \boldsymbol{g}$.
Here the magnetic field strength is normalised by the factor $\sqrt{4 \pi}$. If the magnetic field is prescribed, Eq. (3) splits into a system of first-order partial differential equations describing the horizontal and vertical pressure and density variations from their equilibrium values. These equations are then integrated numerically. This procedure of integration gives satisfactory results in terms of numerical precision.

We use the standard model of the solar photosphere (Spruit 1974) as the unperturbed background model. The unperturbed pressure is recalculated from the standard density profile using the hydrostatic equilibrium condition $\nabla p_{u}=\rho_{u} g$. Then the perturbations to the pressure $p$ and density $\rho$, obtained from the solution of Eq. (3), are added to the unperturbed density and pressure dependencies.

Partial ionisation effects are taken into account when the plasma internal energy and temperature are calculated. The system of Saha equations is solved for the eleven most abundant elements in the solar photosphere to produce the tabulated functions of internal energy $e=e(\rho, p)$ and temperature $T=T(\rho, p)$. Values of internal energy and temperature are then obtained by interpolation. The density, pressure, internal energy and temperature structures are shown in Fig. 3, where the lower right panel shows a significant temperature increase above the MBP. This temperature increase is caused by the increased magnetic tension in the upper layers. A similar, but somewhat weaker, temperature increase is observed in the dynamic radiative magnetoconvection simulations.

For a direct comparison of the radiative properties of the model with observations, we need to know the position of the continuum formation at some wavelength relative to the equipartition layer $\left(v_{\mathrm{A}}=c_{\mathrm{s}}\right)$. The thermal and magnetic structure of the average MBP is shown in the lower right panel of Fig. 3, along with the equipartition layer and continuum formation level $\log \tau_{4300}=0$. The latter curve demonstrates the presence of Wilson depression of the order of $100 \mathrm{~km}$ in the magnetised region, similar to that observed for sunspots. The Wilson depression in the MBP model is significantly less than that of sunspots (Solanki 1993; Watson et al. 2009). The equipartition layer, as is evident from the figure, is located deeper than the continuum formation layer in the region of the strongest magnetic field. This means that in the MBP centre, the $4300 \AA$ continuum is originating from strongly magnetised plasma with $v_{\mathrm{A}}>c_{\mathrm{s}}$ and $\beta<1$, and a variety of MHD effects, including the magneto-acoustic mode conversion, may be observable at this wavelength.

\section{Radiative diagnostics}

The line profile simulation code STOPRO (Solanki 1987; Frutiger 2000; Berdyugina et al. 2003; Shelyag et al. 2004, 2007a) is used to perform the line profile calculations. This code is designed to compute wavelength-dependent intensities and normalised full Stokes vectors for the atomic and molecular line profiles in the LTE approximation. We use STOPRO to calculate the continuum intensities at $4300 \AA$ and $5000 \AA$, as well as G-band intensities for each of the 200 vertical rays of the MBP model described in Sect. 2. To calculate these we employ a procedure similar to that described in detail in Shelyag et al. (2004). The G-band intensities are derived by convolving the simulated spectrum with a $10 \AA$ filter, centered at $4305 \AA$, and integrating this over the range $4295 \AA-4315 \AA$. The intensities are shown in Fig. 4, and have been normalised by the values taken in the nonmagnetic part of the domain corresponding to granulation. As is evident from the figure, the MBP model appears to be bright in 
S. Shelyag et al.: A photospheric bright point model
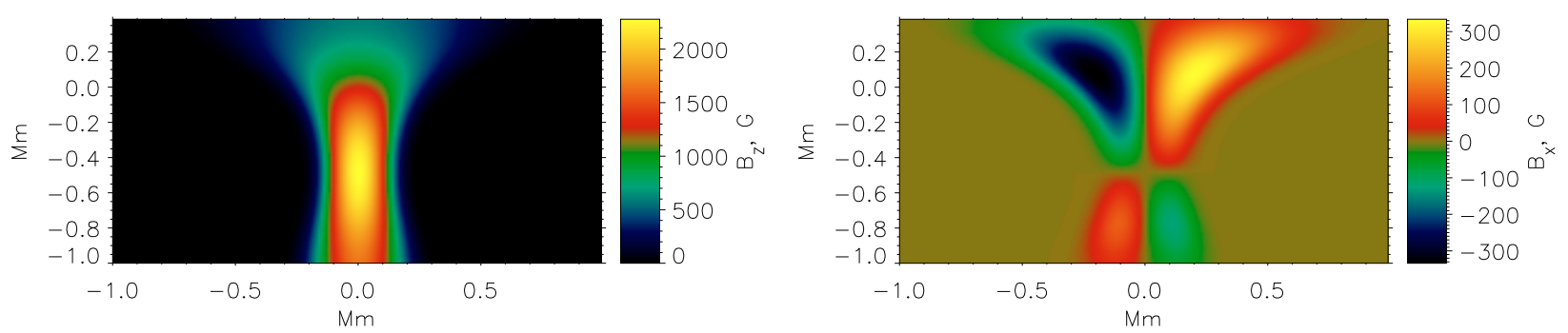

Fig. 2. Vertical (left) and horizontal (right) components of the reconstructed magnetic field for the MBP model.
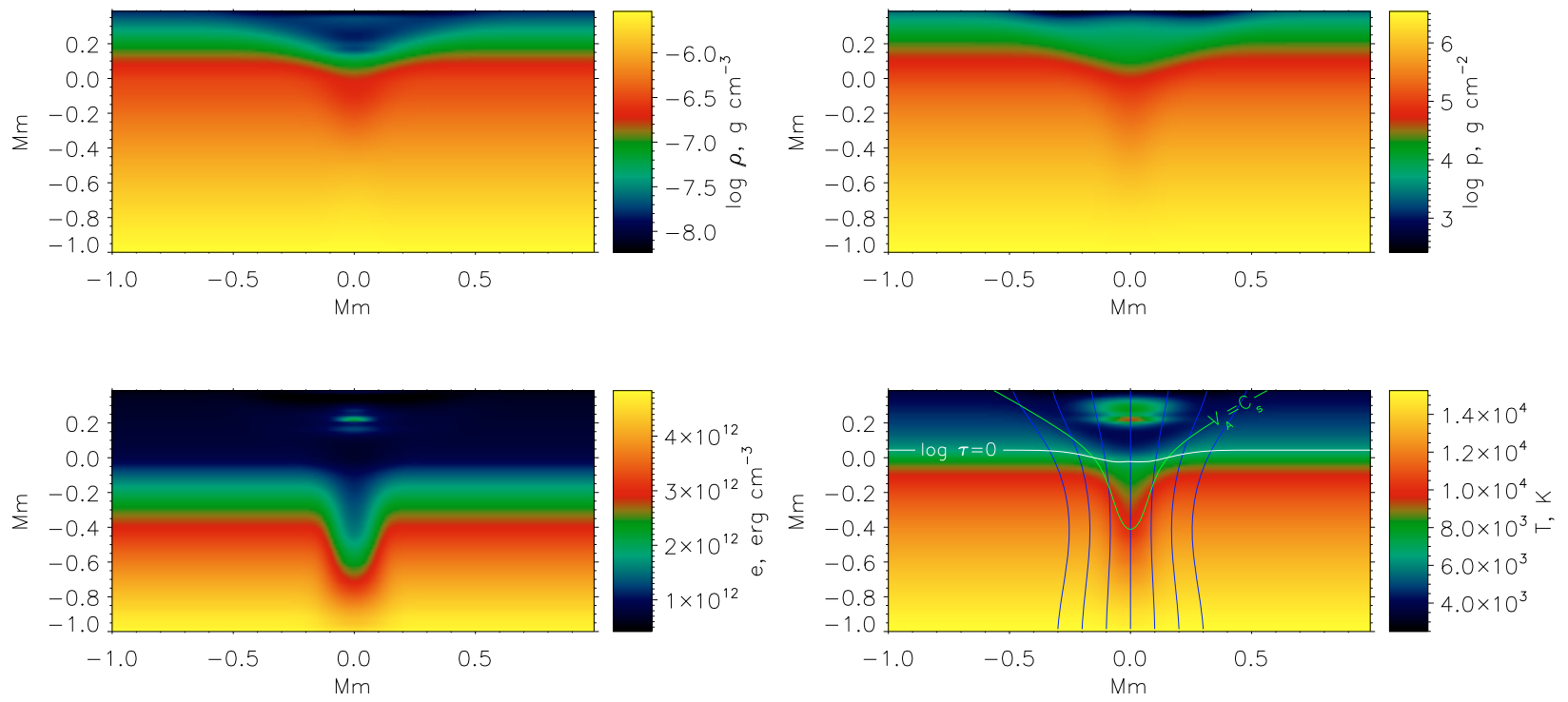

Fig. 3. The theoretical density (top left), pressure (top right), internal energy per unit volume (bottom left) and temperature (bottom right) structures of the MBP. In the bottom right panel, the white line shows the $4300 \AA$ continuum formation level $\log \tau_{4300}=0$, the green line represents the equipartition layer $v_{\mathrm{A}}=c_{\mathrm{s}}$, and the blue lines are the magnetic field lines.

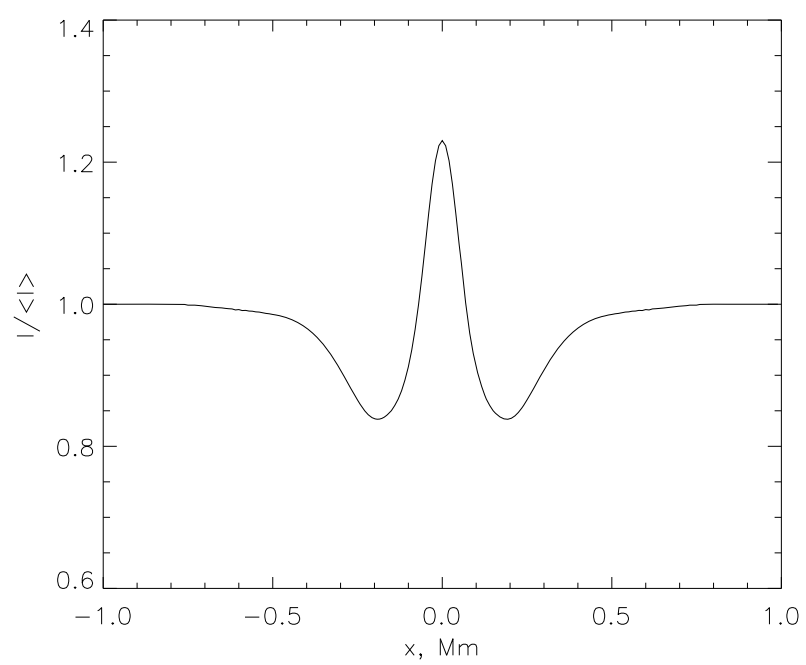

Fig. 4. G-band intensities for the average MBP model. The darkenings, corresponding to the integranular lanes, are found at $x=-0.3 \mathrm{Mm}$ and $x=0.3 \mathrm{Mm}$.

its middle $x=0$. Darkenings, corresponding to the integranular lanes surrounding the MBPs, are found at $x=-0.3 \mathrm{Mm}$ and $x=0.3 \mathrm{Mm}$.

The $6302.49 \AA$ Fe I transition is often used as a diagnostic for the photospheric magnetic field, and the Stokes-I and $-V$ profiles for this line are shown in Fig. 5 for two positions in the model. The $I$ profile is split by strong magnetic field in

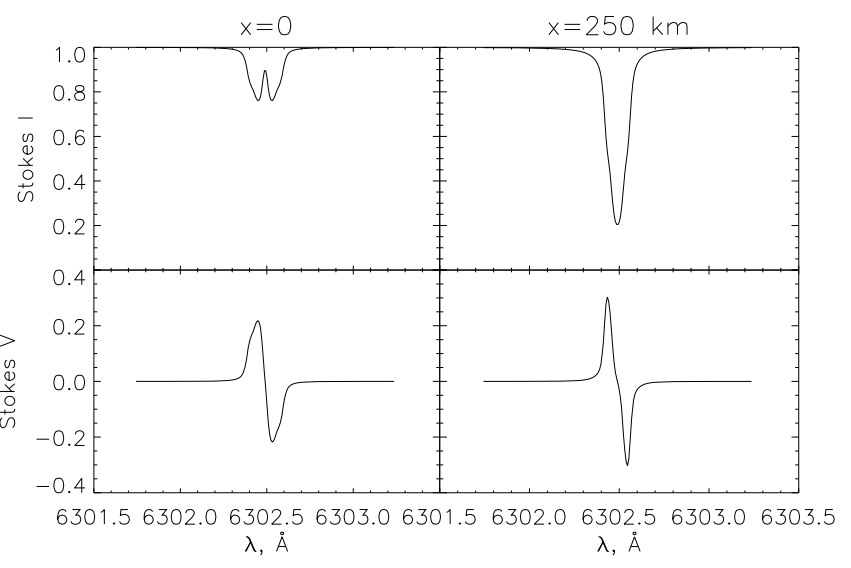

Fig. 5. Stokes- $I$ (top) and $-V$ (bottom) profiles calculated for magnetic flux concentration at $x=0$, (left), and $x=250 \mathrm{~km}$, (right).

the centre of the MBP, but the depth of the profile is reduced due to a smaller temperature gradient. In the weaker magnetic field (Fig. 5, right column), the absorption is stronger, and the Stokes- $V$ profile has a larger amplitude. However, the wavelength separation between the left and right lobes of Stokes- $V$ remain approximately the same in both cases. It should be pointed out that the observational profiles are also influenced by the Doppler shift, caused by the bulk motions of the convecting plasma. Our model does not account for convective velocities 


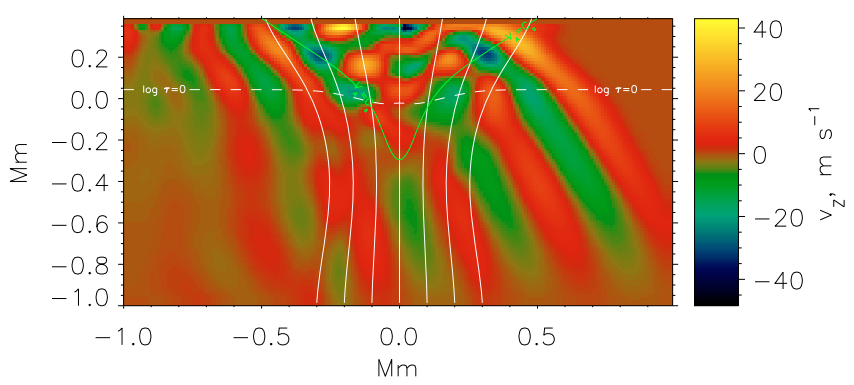

Fig. 6. Vertical velocity component in the domain. Lines as in lower right panel of Fig. 3. A complicated velocity pattern, produced by the interaction of the oncoming wave with the magnetic field concentration, is visible above the equipartition layer $v_{\mathrm{A}}=c_{\mathrm{s}}$.

and thus cannot reproduce the exact central wavelength and $\mathrm{C}$-shape of the observed absorption line profiles.

\section{Wave propagation in the MBP}

We use the code SAC to perform simulations of wave propagation and mode conversion in the MBP (Shelyag et al. 2009; Fedun et al. 2009). The code solves the full ideal MHD equations in a two- or three-dimensional Cartesian grid, and has previously been used for a variety of problems in the physics of solar oscillations. The boundary conditions implemented in the code are "transparent" conditions of the simplest type. These conditions lead to an artificial reflection from the boundary layers, however, for well-resolved perturbations the amplitudes of reflected waves are quite small. The full description of the methods used, boundary conditions and tests are presented in detail by Shelyag et al. (2008). For the simulations, the code has been modified to incorporate the equation of state and account for partial ionisation processes. At each time step, the local internal energy- and density-dependent adiabatic index $\Gamma_{1}$ is calculated using the precalculated tabulated function $\Gamma_{1}=\Gamma_{1}(\rho, e)$. The adiabatic index is then used in the equation of state to relate the kinetic pressure to the internal energy values, thus closing the system of MHD equations.

Oscillations are generated using a single temporally continuous acoustic source located in the non-magnetic part of the numerical domain. This source is coherent, acts in the horizontal direction $v_{x}$, extents vertically over the whole height of the domain, and its period is $T=30 \mathrm{~s}$. The amplitude of the source is $100 \mathrm{~m} \mathrm{~s}^{-1}$, is constant over the domain depth and is chosen to be sufficiently small to keep the oscillations in the linear regime. The diffusive damping introduced into the code by increasing the hyperdiffusion coefficients to 0.2 , combined with the low source amplitude, prevent the convectively unstable domain from going into the convective regime for a sufficiently long time.

A snapshot of the vertical velocity component, taken at $t=$ $160 \mathrm{~s}$ from the start of the simulation, is shown in Fig. 6. An oscillatory pattern, bounded by the equipartition level $v_{\mathrm{A}}=c_{\mathrm{S}}$ (marked by the green line in the figure), is easily distinguishable. This pattern is produced by an interference of the slow and fast magneto-acoustic waves, propagating differently in the region. Most of the velocity pattern is above the continuum formation level $\log \tau=0$ (white dashed line), and should therefore affect the absorption line profiles.

Figure 7 shows the $5000 \AA$ continuum intensity variations in non-magnetic media outside the MBP (dashed lines) and in the centre of the MBP (solid lines). It is evident from the figure that both the absolute intensity variation (upper plot) and the

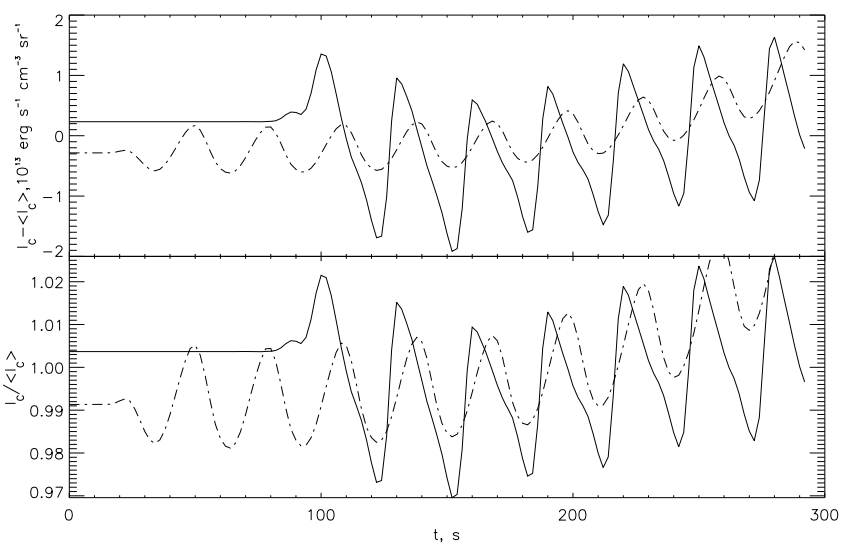

Fig. 7. Absolute (upper plot) and relative (lower plot) continuum intensity oscillations in the non-magnetic media $(x=0.66 \mathrm{Mm}$, dashed line) and in the bright point centre $(x=0$, solid line).

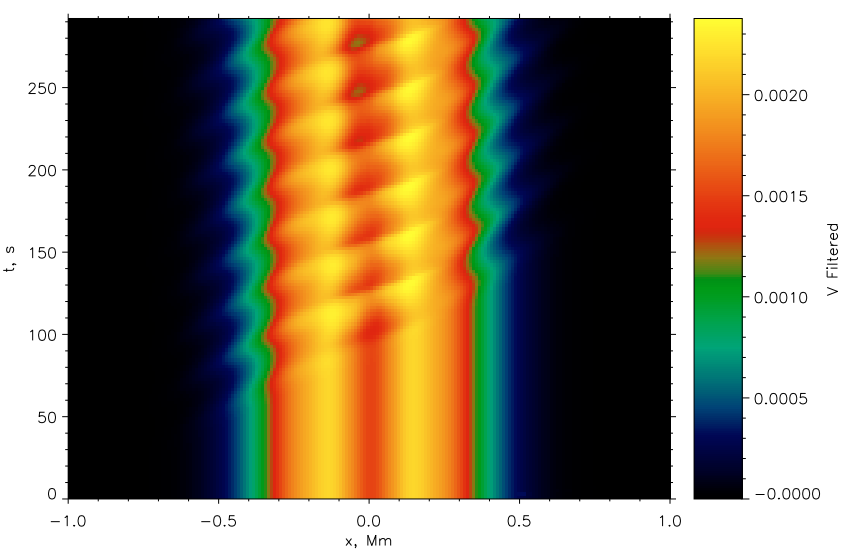

Fig. 8. Filtered Stokes- $V$ intensities, calculated for the simulated time series.

intensity variation normalised to the average continuum intensity (lower plot), are larger in the MBP than in non-magnetic media. This is caused by the larger continuum intensity and stronger variation of temperature in the deeper (due to depression) layers of the solar photosphere in the MBP. Although the source amplitude is sufficiently low to keep the linear character of the intensity oscillations in the non-magnetic part of the domain (dashed lines), a non-linear character of the intensity oscillations in the MBP is also observed (solid lines). The steepening of the wave front observed in the figure is caused by conversion of the linear wave into the non-linear regime in the partially evacuated plasma of the MBP.

An oscillatory behaviour can also be detected in the magnetic field. To reveal the observational consequences of the oscillations in the magnetic field, we apply the standard observational technique of measuring the Stokes- $V$ profile. We choose a narrow bandpass filter with a width of $0.02 \AA$, centered $-0.0525 \AA$ from the Fe I line centre. We then apply this filter to the Stokes- $V$ profiles data, calculated for the whole $x-t$ domain. The resultant Stokes- $V$ intensities are shown in Fig. 8. The vertical component of the magnetic field in the region of the line formation is proportional to the intensity of the Stokes- $V$ filter. However, it is worth noting that the Stokes- $V$ profile for the $6302 \AA$ line saturates in regions of strong magnetic field. As Fig. 8 demonstrates, the intensity at $x=0$ is equal to the intensity at about $\pm 0.3 \mathrm{Mm}$. Also, the magnetic field strength values, restored from the Stokes- $V$ intensities at these positions, are virtually indistinguishable, thus 


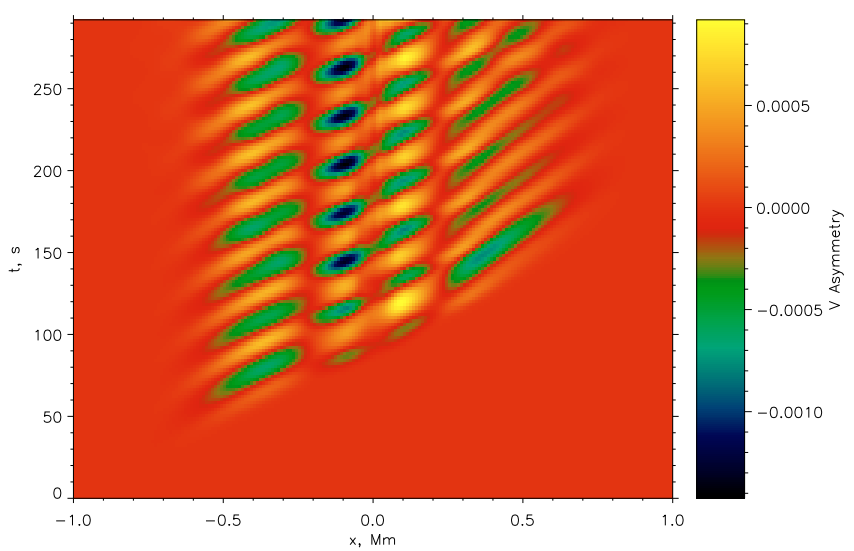

Fig. 9. Stokes- $V$ asymmetries, calculated for the simulated time series.

making the $6302.49 \AA \mathrm{Fe}$ I line unsuitable for measurements of strong magnetic fields. The variation in the filtered Stokes $V$ amplitudes in the centre of MBP is about $25-30 \%$ in our simulations. This value is quite large and should therefore be detectable by observations.

The magnetic field configuration in the simulations is localised and non-uniform, and the line-of-sight therefore crosses regions of both zero and non-zero magnetic field. The initial static model does not produce an asymmetry in the Stokes- $V$ profiles, since it has no velocities which are necessary to produce an asymmetry (see e.g. Sankarasubramanian \& Rimmele 2002; Bellot Rubio et al. 2000). In the presence of a flow, caused by the perturbation introduced into the numerical box, this magnetic field configuration can lead to asymmetric Stokes- $V$ profiles (Solanki 1993). The simplest measure of the area asymmetry is the integral of the Stokes- $V$ profile over the wavelength $A=\int V \mathrm{~d} \lambda$. The $V$ profile area asymmetry map is shown in Fig. 9. The normalised amplitude of Stokes- $V$ area asymmetry is of the order of $2 \%$ in the center of the MBP. This value is close to the observed values of Stokes- $V$ area asymmetries, which are about 3\% (Bellot Rubio et al. 2000). Thus, the variation of the Stokes- $V$ area asymmetry produced by the perturbation is large enough to be detected by modern instruments.

\section{Concluding remarks}

We have presented the recipe for creating a static numerical model of a photospheric MBP. This model allows the study of oscillatory properties of such small magnetic configurations in the solar photosphere directly by measuring their radiation intensity and polarimetric properties. The magnetic field is extracted from dynamic simulations of solar radiative magneto-convection and reconstructed using the assumption of self-similarity. Thus, our static model inherits many observational properties of the full dynamic simulation.

The model reproduces the G-band brightening in the MBP centre. Stokes- $I$ and - $V$ profiles for the $6302 \AA$ FeI line are comparable to solar observations in both the magnetised region and in the region corresponding to the non-magnetic solar granulation. The continuum formation level in the centre of the MBP is located in the region of strongly magnetised plasma, where the Alfvén speed is greater than that of sound.

We have used the model to examine the observational consequences of sound wave propagation though the magnetic field concentration of the MBP. This preliminary investigation shows that the variation of continuum intensity is more pronounced in the MBP compared to the average granule. Using the radiative diagnostics with the Stokes- $V$ profile of the $6302 \AA$ Fe I absorption line, we demonstrate the detectability of the magnetic field variation in the bright point and show the appearance of the Stokes- $V$ profile asymmetry caused by the oscillations.

The radiative heating term is not included into the system of MHD equations used to perform the wave dynamics study. The absence of the heating term may result in reduced vertical radiative flux in the magnetic flux tube. As a result, we have somewhat smaller continuum and G-band intensity in the MBPs, compared to the full simulations and the real Sun. However, for wave dynamics the effect of the radiative term is expected to be small. As it has recently been shown by Yelles Chaouche et al. (2009), the magnetic flux tubes in the solar photosphere are "reasonably well reproduced" by a thin flux tube approximation with no radiative term included.

Future investigations will examine the response to sources at different locations and with a range of frequencies. The behaviour and observational signatures of Alfvén waves in small magnetic elements is particularly important when used as a diagnostic for solar plasma parameters, and for understanding the energy transport to the corona. Thus we plan to extend the model to three dimensions.

Acknowledgements. This work has been supported by the UK Science and Technology Facilities Council (STFC). F.P.K. is grateful to AWE Aldermaston for the award of a William Penney Fellowship.

\section{References}

Bellot Rubio, L. R., Ruiz Cobo, B., \& Collados, M. 2000, ApJ, 535, 489 Berdyugina, S. V., Solanki, S. K., \& Frutiger, C. 2003, A\&A, 412, 513 Carlsson, M., Stein, R. F., Nordlund, Å., \& Scharmer, G. B. 2004, ApJ, 610, L137

Fedun, V., Erdélyi, R., \& Shelyag, S. 2009, Sol. Phys., 258, 219

Frutiger, C. 2000, Ph.D. Thesis No. 13896, ETH Zürich

Gordovskyy, M., \& Jain, R. 2007, ApJ, 661, 586

Hanasoge, S. M., Duvall, T. L., Jr., \& Couvidat, S. 2007, ApJ, 664, 1234

Hasan, S. S., \& van Ballegooijen, A. A. 2008, ApJ, 680, 1542

Hasan, S. S., van Ballegooijen, A. A., Kalkofen, W., \& Steiner, O. 2005, ApJ, 631,1270

Khomenko, E., \& Collados, M. 2006, ApJ, 653, 739

Khomenko, E., \& Collados, M. 2009, A\&A, 506, L5

Khomenko, E., Kosovichev, A., Collados, M., Parchevsky, K., \& Olshevsky, V. 2009, ApJ, 694, 411

Parchevsky, K. V., \& Kosovichev, A. G. 2009, ApJ, 694, 573

Sankarasubramanian, K., \& Rimmele, T. 2002, ApJ, 576, 1048

Schlüter, A., \& Temesváry, S. 1958, Electromagnetic Phenomena in Cosmical Physics, 6, 263

Schüssler, M., Shelyag, S., Berdyugina, S., Vögler, A., \& Solanki, S. K. 2003, ApJ, 597, L173

Schüssler, M., \& Rempel, M. 2005, A\&A, 441, 337

Shelyag, S., Schüssler, M., Solanki, S. K., Berdyugina, S. V., \& Vögler, A. 2004, A\&A, 427, 335

Shelyag, S., Schüssler, M., Solanki, S. K., \& Vögler, A. 2007a, A\&A, 469, 731

Shelyag, S., Erdélyi, R., \& Thompson, M. J. 2007b, A\&A, 469, 1101

Shelyag, S., Fedun, V., \& Erdélyi, R. 2008, A\&A, 486, 655

Shelyag, S., Zharkov, S., Fedun, V., Erdélyi, R., \& Thompson, M. J. 2009, A\&A, 501,735

Solanki, S. K. 1987, Ph.D. Thesis No8309, ETH Zürich

Solanki, S. K. 1993, Space Sci. Rev., 63, 1

Solanki, S. K. 2003, A\&ARv, 11, 153

Spruit, H. C. 1974, Sol. Phys., 34, 277

Steiner, O. 2009 [arXiv: 0904 . 2026]

Vigeesh, G., Hasan, S. S., \& Steiner, O. 2009, A\&A 508, 951

Vögler, A., Shelyag, S., Schüssler, M. et al. 2005, A\&A, 429, 335

Wilson, A., \& Maskelyne, N. 1774, Royal Soc. London Philos. Trans. Ser. I, 64, 1

Watson, F., Fletcher, L., Dalla, S., \& Marshall, S. 2009, Sol. Phys., 260, 5

Yelles Chaouche, L., Solanki, S. K., \& Schüssler, M. 2009, A\&A, 504, 595 